\title{
New physics search in the LHCb era
}

\author{
Tobias Hurth
}

CERN, Theory Group, CH-1211 Geneva 23, Switzerland

SLAC, Stanford University, Stanford, CA 94309, USA

DOI: http://dx.doi.org/10.3204/DESY-PROC-2009-01/85

\begin{abstract}
We present theoretical and experimental preparations for an indirect search for new physics (NP) using the rare decay $\bar{B}_{d} \rightarrow \bar{K}^{* 0} \mu^{+} \mu^{-}$. We design new observables with very small theoretical uncertainties and good experimental resolution.
\end{abstract}

\section{Introduction}

At the start of the LHC we are confronted with the experimental fact that all data on flavour observables from Babar, Belle, CLEO and also from D0 and CDF are consistent with the Standard Model (SM) predictions [1]. This implies that generic new physics (NP) contributions in $K-\bar{K}$ mixing for example guide us to a new-physics scale of $10^{3}-10^{4} \mathrm{TeV}$ depending if the new contributions enter at loop- or tree-level. This is in strong contrast to the working hypothesis of the LHC that there is NP "around the corner" at $1 \mathrm{TeV}$ in order to stabilise the Higgs boson mass. Therefore, any NP at the $1 \mathrm{TeV}$ scale has to have a non-generic flavour structure and we have to understand why new flavour-changing neutral currents (FCNC) are suppressed. Rare decays and $\mathrm{CP}$ violating observables allow an analysis of this flavour problem.

The crucial problem in the new physics search within flavour physics is the optimal separation of NP effects from hadronic uncertainties. It is well known that inclusive decay modes are dominated by partonic contributions; non-perturbative corrections are in general rather small [2, 3]. Also ratios of exclusive decay modes such as asymmetries are well suited for the new-physics search. Here large parts of the hadronic uncertainties partially cancel out; for example, there are $\mathrm{CP}$ asymmetries that are governed by one weak phase only; thus the hadronic matrix elements cancel out completely. It is the latter opportunity which represents the general strategy followed by $\mathrm{LHC} b$ for the construction of theoretically clean observables.

In this letter we briefly discuss the theoretical and experimental preparations for an indirect NP search using the rare decay $\bar{B}_{d} \rightarrow \bar{K}^{* 0} \mu^{+} \mu^{-}$based on the QCDf/SCET approach [4]. QCD corrections are included at the next-to-leading order level and also the impact of the unknown $\Lambda / m_{b}$ corrections is made explicit.

The exclusive decay $\bar{B}_{d} \rightarrow \bar{K}^{* 0} \mu^{+} \mu^{-}$was first observed at Belle [5]. It offers a rich phenomenology of various kinematic distributions beyond the measurement of the branching ratio. We note that some experimental analyses of those angular distributions are already presented by the $B$ factories $[6,7,9,10]$. Those experimental results already have a significant impact on the model-independent constraints within the minimal flavour violation approach [8].

Large increase in statistics at $\mathrm{LHC} b[11-13]$ for $\bar{B}_{d} \rightarrow \bar{K}^{* 0} \mu^{+} \mu^{-}$will make much higher precision measurements possible. There are also great opportunities at the future (Super-) $B$ 
factories in this respect [14-17].

Previously proposed angular distributions and $C P$ violating observables in $\bar{B}_{d} \rightarrow \bar{K}^{* 0} \mu^{+} \mu^{-}$ are reviewed in Ref. [23], and more recently QCDf analyses of such angular distributions [24,25] and CP violating observables [26], based on the NLO results in Ref. [27], were presented.

\section{QCD factorization, SCET}

Regarding the hadronic matrix elements of exclusive modes, the method of QCD-improved factorization (QCDf) has been systemized for non-leptonic decays in the heavy-quark limit. This method allows for a perturbative calculation of QCD corrections to naive factorization and is the basis for the up-to-date predictions for exclusive rare $B$ decays in general [18].

A quantum field theoretical framework was proposed - known under the name of softcollinear effective field theory (SCET) - which allows for a deeper understanding of the QCDf approach $[19,20]$. In contrast to the heavy-quark effective theory (HQET), SCET does not correspond to a local operator expansion. HQET is only applicable to $B$ decays, when the energy transfer to light hadrons is small, for example to $B \rightarrow D$ transitions at small recoil to the $D$ meson. HQET is not applicable, when some of the outgoing, light particles have momenta of order $m_{b}$; then one faces a multi scale problem that can be tackled within SCET.

There are three scales: a) $\Lambda=$ few $\times \Lambda_{\mathrm{QCD}}$ the soft scale set by the typical energies and momenta of the light degrees of freedom in the hadronic bound states; b) $m_{b}$ the hard scale set by the heavy- $b$-quark mass and also by the energy of the final-state hadron in the $B$-meson rest frame; and c) the hard-collinear scale $\mu_{\mathrm{hc}}=\sqrt{m_{b} \Lambda}$ appears through interactions between soft and energetic modes in the initial and final states. The dynamics of hard and hard-collinear modes can be described perturbatively in the heavy-quark limit $m_{b} \rightarrow \infty$. Thus, SCET describes $B$ decays to light hadrons with energies much larger than their masses, assuming that their constituents have momenta collinear to the hadron momentum.

However, we emphasize that within the QCDf/SCET approach, a general, quantitative method to estimate the important $\Lambda / m_{b}$ corrections to the heavy-quark limit is missing which has important phenomenological consequences.

A careful choice of observables needs to be made to take full advantage of the exclusive decay $\bar{B}_{d} \rightarrow \bar{K}^{* 0} \mu^{+} \mu^{-}$, as only in certain ratios such as $C P$ and forward-backward asymmetries, the hadronic uncertainties cancel out making such ratios the only observables that are highly sensitive to NP.

Within the QCDf/SCET approach one finds crucial form factor relations [21] which simplify the theoretical structure of various kinematical distributions such that, at least at the leading order (LO) level any hadronic uncertainties cancel out. A well-known example of this is the zero-crossing of the forward-backward asymmetry. In [4] new observables of this kind in the $\bar{B}_{d} \rightarrow \bar{K}^{* 0} \mu^{+} \mu^{-}$decay were proposed which have very small theoretical uncertainties and good experimental resolution. The only difference to the forward-backward asymmetry is that within these new observables the hadronic form factors cancel out for all values of the dilepton mass. 


\section{Theoretical preliminaries}

The decay $\bar{B}_{d} \rightarrow \bar{K}^{* 0} \ell^{+} \ell^{-}$with $\bar{K}^{* 0} \rightarrow K^{-} \pi^{+}$on the mass shell is completely described by four independent kinematic variables, the lepton-pair invariant mass squared, $q^{2}$, and the three angles $\theta_{l}, \theta_{K}, \phi$. Summing over the spins of the final particles, the differential decay distribution of $\bar{B}_{d} \rightarrow \bar{K}^{* 0} \ell^{+} \ell^{-}$can be written as [28-31]:

$$
\frac{d^{4} \Gamma_{\bar{B}_{d}}}{d q^{2} d \theta_{l} d \theta_{K} d \phi}=\frac{9}{32 \pi} I\left(q^{2}, \theta_{l}, \theta_{K}, \phi\right) \sin \theta_{l} \sin \theta_{K}
$$

with

$$
\begin{aligned}
I= & I_{1}+I_{2} \cos 2 \theta_{l}+I_{3} \sin ^{2} \theta_{l} \cos 2 \phi+I_{4} \sin 2 \theta_{l} \cos \phi+I_{5} \sin \theta_{l} \cos \phi \\
& +I_{6} \cos \theta_{l}+I_{7} \sin \theta_{l} \sin \phi+I_{8} \sin 2 \theta_{l} \sin \phi+I_{9} \sin ^{2} \theta_{l} \sin 2 \phi .
\end{aligned}
$$

The $I_{i}$ depend on products of the seven complex $K^{*}$ spin amplitudes, $A_{\perp L / R}, A_{\| L / R}, A_{0 L / R}, A_{t}$ with each of these a function of $q^{2}$; the explicit formulae are given in the appendix. $A_{t}$ is related to the time-like component of the virtual $K^{*}$, which does not contribute in the case of massless leptons and can be neglected if the lepton mass is small in comparison to the mass of the lepton pair. We will consider this case in our present analysis.

The six complex $K^{*}$ spin amplitudes of the massless case are related to the well-known helicity amplitudes (used for example in [29, 30,32]):

$$
A_{\perp, \|}=\left(H_{+1} \mp H_{-1}\right) / \sqrt{2}, \quad A_{0}=H_{0} .
$$

The crucial theoretical input we use in our analysis is the observation that in the limit where the initial hadron is heavy and the final meson has a large energy [21] the hadronic form factors can be expanded in the small ratios $\Lambda_{\mathrm{QCD}} / m_{b}$ and $\Lambda_{\mathrm{QCD}} / E$, where $E$ is the energy of the light meson. Neglecting corrections of order $1 / m_{b}$ and $\alpha_{s}$, the seven a priori independent $B \rightarrow K^{*}$ form factors reduce to two universal form factors $\xi_{\perp}$ and $\xi_{\|}[21,22]$ and one finds that the spin amplitudes at leading order in $1 / m_{b}$ and $\alpha_{s}$ have a very simple form:

$$
\begin{aligned}
& A_{\perp L, R}=\sqrt{2} N m_{B}(1-\hat{s}) \times\left[\left(\mathcal{C}_{9}^{(\mathrm{eff})} \mp \mathcal{C}_{10}\right)+\frac{2 \hat{m}_{b}}{\hat{s}}\left(\mathcal{C}_{7}^{(\mathrm{eff})}+\mathcal{C}_{7}^{(\mathrm{eff})}\right)\right] \xi_{\perp}\left(E_{K^{*}}\right) \\
& A_{\| L, R}=-\sqrt{2} N m_{B}(1-\hat{s}) \times\left[\left(\mathcal{C}_{9}^{(\mathrm{eff})} \mp \mathcal{C}_{10}\right)+\frac{2 \hat{m}_{b}}{\hat{s}}\left(\mathcal{C}_{7}^{(\mathrm{eff})}-\mathcal{C}_{7}^{\prime(\mathrm{eff})}\right)\right] \xi_{\perp}\left(E_{K^{*}}\right) \\
& A_{0 L, R}=-\frac{N m_{B}}{2 \hat{m}_{K^{*}} \sqrt{\hat{s}}}(1-\hat{s})^{2}\left[\left(\mathcal{C}_{9}^{(\mathrm{eff})} \mp \mathcal{C}_{10}\right)+2 \hat{m}_{b}\left(\mathcal{C}_{7}^{(\mathrm{eff})}-\mathcal{C}_{7}^{\prime(\mathrm{eff})}\right)\right] \xi_{\|}\left(E_{K^{*}}\right),
\end{aligned}
$$

with $\hat{s}=q^{2} / m_{B}^{2}, \hat{m}_{i}=m_{i} / m_{B}$. Here we neglected terms of $O\left(\hat{m}_{K^{*}}^{2}\right)$. It is important to mention that the theoretical simplifications are restricted to the kinematic region in which the energy of the $K^{*}$ is of the order of the heavy quark mass, i.e. $q^{2} \ll m_{B}^{2}$. Moreover, the influences of very light resonances below $1 \mathrm{GeV}$ question the $\mathrm{QCD}$ factorization results in that region. Thus, we will confine our analysis of all observables to the dilepton mass in the range $1 \mathrm{GeV}^{2} \leq q^{2} \leq$ $6 \mathrm{GeV}^{2}$. 


\section{Construction of theoretically clean observables}

By inspection one finds that the distribution functions $I_{i}$ in the differential decay distribution (see Eq. (10)) are invariant under three symmetry transformations which are given explicitly in the appendix (see Eqs. (11-13)). This implies that only 9 of the $12 K^{*}$ spin amplitudes are independent and that they can be fixed by an full angular fit to the 9 independent coefficients of the differential decay distribution. Another direct consequence is that any observable based on the differential decay distribution has also to be invariant under the same symmetry transformations.

Besides this mandatory criterium there are further criteria required for an interesting observable. [Simplicity:] A simple functional dependence on the 9 independent measurable distribution functions; at best it should depend only from one or two in the numerator and denominator of an asymmetry. [Cleanliness:] At leading order in $\Lambda / m_{b}$ and in $\alpha_{s}$ the observable should be independent of any form factor, at best for all $q^{2}$. Also the influence of symmetry-breaking corrections at order $\alpha_{s}$ and at order $\Lambda / m_{b}$ should be minimal. [Sensitivity:] The sensitivity to the $\mathcal{C}_{7}^{\prime}$ (eff) Wilson coefficient representing NP with another chirality than in the SM should be maximal. [Precision:] The experimental precision obtainable should be good enough to distinguish different NP models.

In the limit where the $\bar{K}^{* 0}$ meson has a large energy, only two independent form factors occur in $A_{0 L / R}$ and in $A_{\perp L / R}$ and $A_{\| L / R}$. Clearly, any ratio of two of the nine measurable distribution functions proportional to the same form factor fulfil the criterium of symmetry, simplicity, and theoretical cleanliness up to $\Lambda / m_{b}$ and $\alpha_{s}$ corrections. However, the third criterium, a sensitivity to a special kind of NP and the subsequent requirement of experimental precision, singles out particular combinations. In [4] we focused on new right-handed currents. Other NP sensitivities may single out other observables as will be analysed in a forthcoming paper [33].

\section{Results}

The first surprising result is that the previously proposed quantity $A_{T}^{(1)}$ [29],

$$
A_{T}^{(1)}=\frac{\Gamma_{-}-\Gamma_{+}}{\Gamma_{-}+\Gamma_{+}}=\frac{-2 \Re\left(A_{\|} A_{\perp}^{*}\right)}{\left|A_{\perp}\right|^{2}+\left|A_{\|}\right|^{2}} .
$$

with $\Gamma_{ \pm}=\left|H_{ \pm 1}^{L}\right|^{2}+\left|H_{ \pm 1}^{R}\right|^{2}$ does not fulfil the most important criterium of symmetry while it has very attractive new physics sensitivity $[24,25]$. Therefore, it is not possible to extract $A_{T}^{(1)}$ from the full angular distribution which is constructed after summing over the spins of the final particles. Because it seems practically not possible to measure the helicity of the final states on

a event-by-event basis, $A_{T}^{(1)}$ cannot be measured at either LHC $b$ or at a Super- $B$ factory with electrons or muons in the final state.

One finds that the well-known quantities, the forward-backward asymmetry $A_{\mathrm{FB}}$ and the longitudinal $K^{*}$ polarization $F_{L}$ fulfill the symmetry but they include larger theoretical uncertainties due to the fact that the form factors do not cancel at leading order level for all dilepton masses. Moreover, the sensitivity to right-handed currents is marginal as it is shown below,

$$
A_{\mathrm{FB}}=\frac{3}{2} \frac{\Re\left(A_{\| L} A_{\perp L}^{*}\right)-\Re\left(A_{\| R} A_{\perp R}^{*}\right)}{\left|A_{0}\right|^{2}+\left|A_{\|}\right|^{2}+\left|A_{\perp}\right|^{2}}
$$



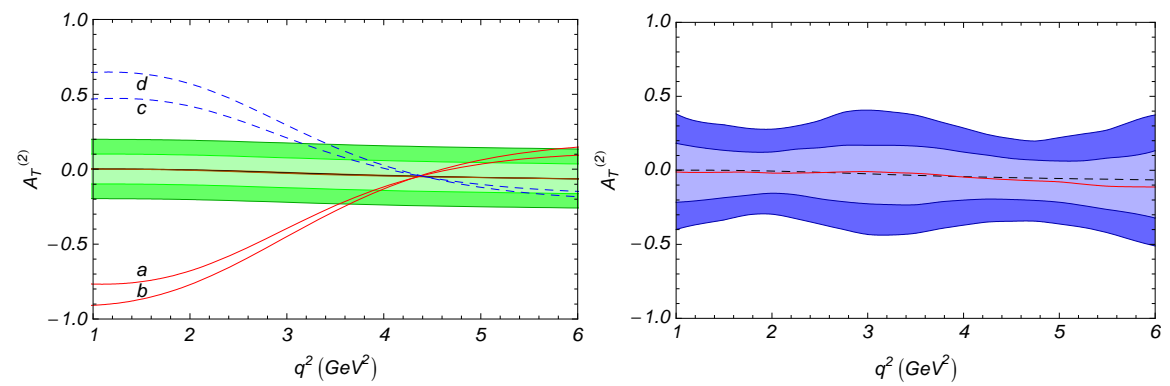

Fig. 1: For $A_{T}^{(2)}$, theoretical errors (top), experimental errors (bottom) as a function of the squared dimuon mass, see text for details.
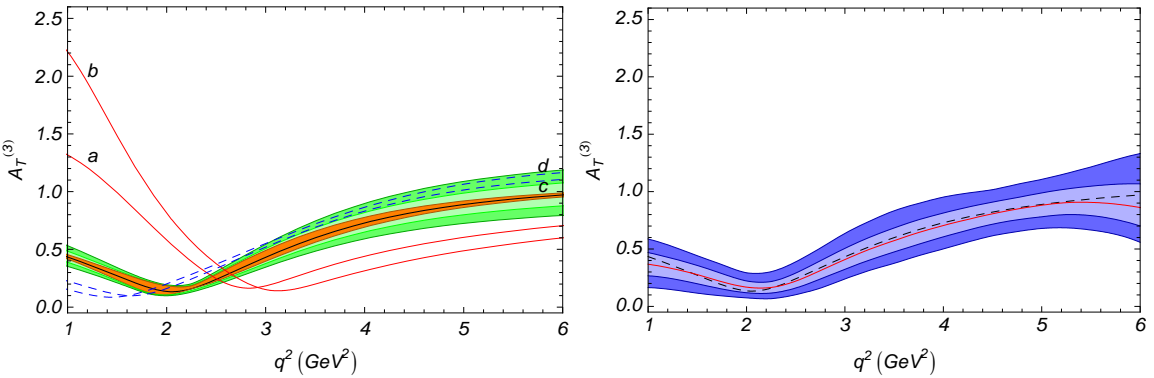

Fig. 2: $A_{T}^{(3)}$, as in Fig.1.
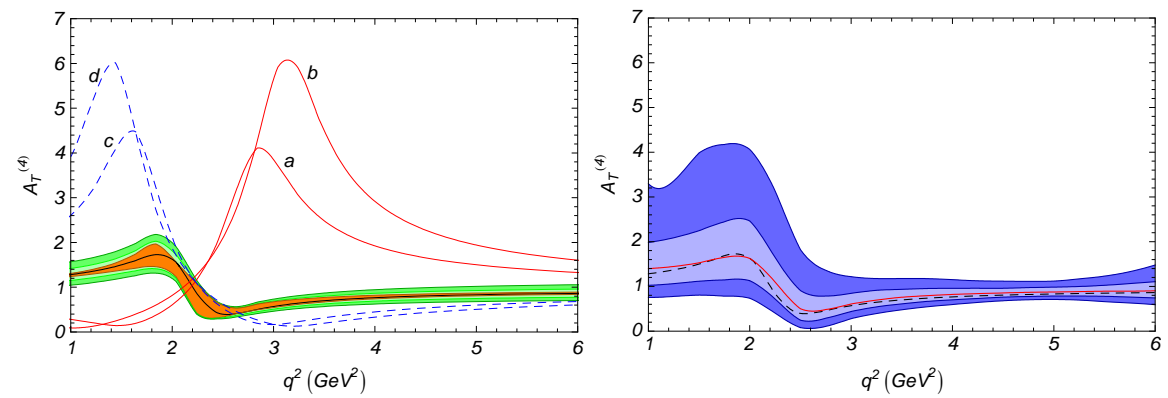

Fig. 3: $A_{T}^{(4)}$, as in Fig.1.
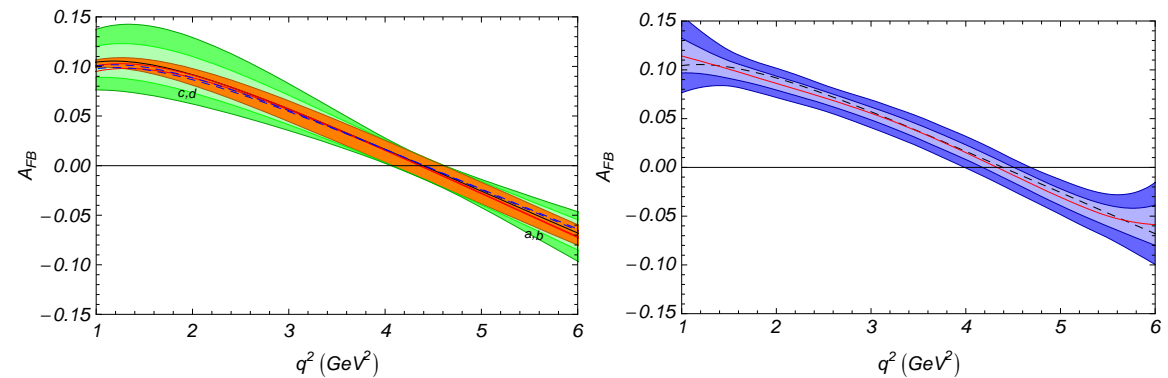

Fig. 4: $A_{F B}$, as in Fig.1. 

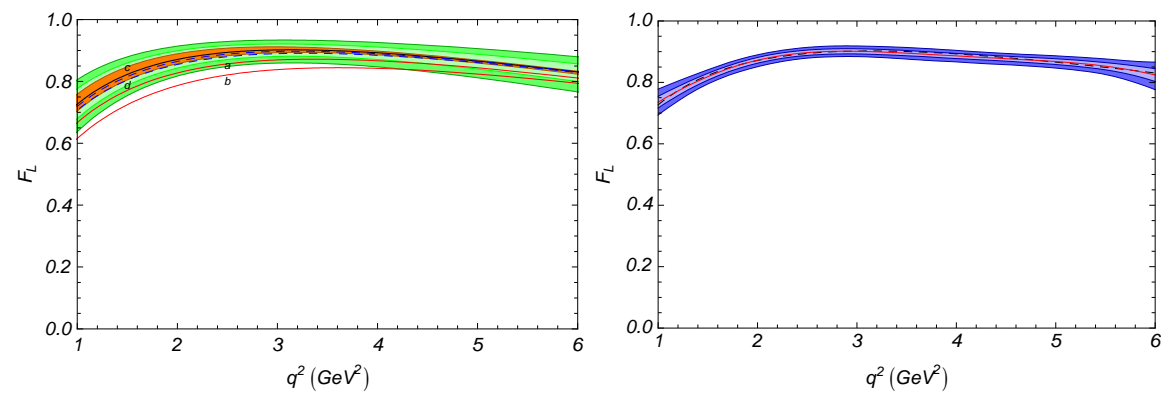

Fig. 5: $F_{L}$, as in Fig.1.

where for $i, j=0, \|, \perp$

$$
\begin{gathered}
A_{i} A_{j}^{*} \equiv A_{i L}\left(q^{2}\right) A_{j L}^{*}\left(q^{2}\right)+A_{i R}\left(q^{2}\right) A_{j R}^{*}\left(q^{2}\right), \\
F_{L}\left(q^{2}\right)=\frac{\left|A_{0}\right|^{2}}{\left|A_{0}\right|^{2}+\left|A_{\|}\right|^{2}+\left|A_{\perp}\right|^{2}} .
\end{gathered}
$$

In contrast, the following three observables,

$$
\begin{gathered}
A_{T}^{(2)}=\frac{\left|A_{\perp}\right|^{2}-\left|A_{\|}\right|^{2}}{\left|A_{\perp}\right|^{2}+\left|A_{\|}\right|^{2}}, \quad A_{T}^{(3)}=\frac{\left|A_{0 L} A_{\| L}^{*}+A_{0 R}^{*} A_{\| R}\right|}{\sqrt{\left|A_{0}\right|^{2}\left|A_{\perp}\right|^{2}}}, \\
A_{T}^{(4)}=\frac{\left|A_{0 L} A_{\perp L}^{*}-A_{0 R}^{*} A_{\perp R}\right|}{\left|A_{0 L}^{*} A_{\| L}+A_{0 R} A_{\| R}^{*}\right|},
\end{gathered}
$$

are theoretically clean for all dilepton masses and also show a very high sensitivity to righthanded currents.

In the following figures the results on the observables, $F_{L}, A_{F B}, A_{T}^{(2)}, A_{T}^{(3)}$, and $A_{T}^{(4)}$ are illustrated: For all the observables the theoretical sensitivity is plotted on the top of each figure. The thin dark line is the central NLO result for the SM and the narrow inner dark (orange) band that surrounds it corresponds to the NLO SM uncertainties due to both input parameters and perturbative scale dependence. Light grey (green) bands are the estimated $\Lambda / m_{b} \pm 5 \%$ corrections for each spin amplitude while darker grey (green) ones are the more conservative $\Lambda / m_{b} \pm 10 \%$ corrections. The curves labelled (a)-(d) correspond to four different benchmark points in the MSSM for righthanded currents (for more details see [4]). The experimental sensitivity for a dataset corresponding to $10 \mathrm{fb}^{-1}$ of $\mathrm{LHC} b$ data is given in each figure on the bottom, assuming the SM. Here the solid (red) line shows the median extracted from the fit to the ensemble of data and the dashed (black) line shows the theoretical input distribution. The inner and outer bands correspond to $1 \sigma$ and $2 \sigma$ experimental errors.

The observables $A_{T}^{(3)}$ and $A_{T}^{(4)}$ offer sensitivity to the longitudinal spin amplitude $A_{0 L, R}$ in a controlled way compared to the old observable $F_{L}$ : the dependence on both the parallel and perpendicular soft form factors $\xi_{\|}(0)$ and $\xi_{\perp}(0)$ cancels at LO. A residual of this dependence may appear at NLO, but as shown in Figs. 2 and 3, it is basically negligible. It is also remarkable that for $A_{T}^{(3)}$ and $A_{T}^{(4)}$ at low $q^{2}$ the impact of this uncertainty is less important than the 

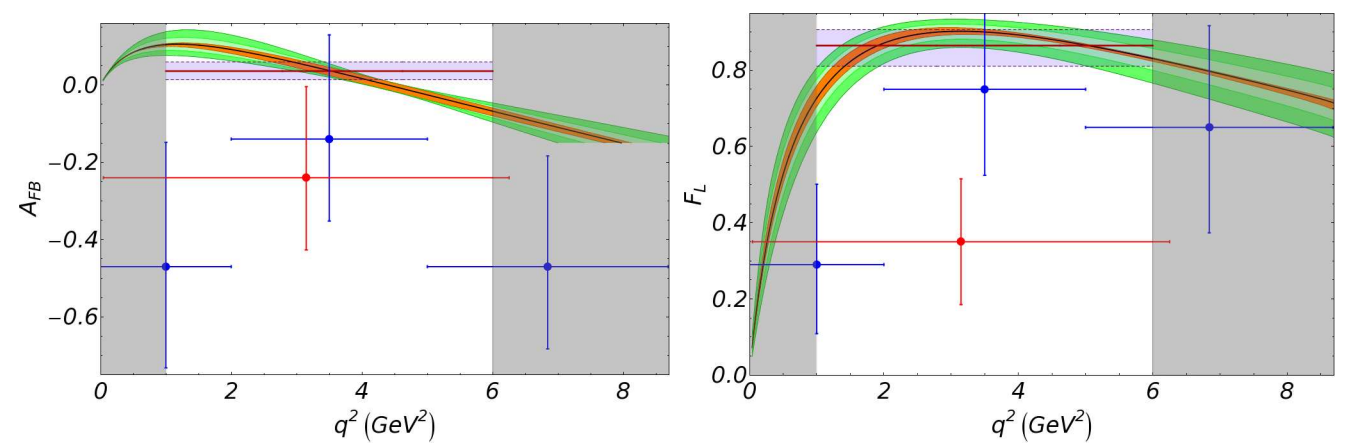

Fig. 6: Belle (black/blue) and BaBar (grey/red) data points on $F_{L}$ and on $A_{F B}$ with SM predictions and weighted SM averages over the bin $q^{2} \in\left[1 \mathrm{GeV}^{2}, 6 \mathrm{GeV}^{2}\right]$

uncertainties due to input parameters and scale dependence. The observables $A_{T}^{(3)}$ and $A_{T}^{(4)}$ also present a different sensitivity to $\mathcal{C}_{7}^{\prime}$ via their dependence on $A_{0 L, R}$ compared with $A_{T}^{(2)}$. This may allow for a particularly interesting cross check of the sensitivity to this chirality flipped operator $\mathcal{O}_{7}^{\prime}$; for instance, new contributions coming from tensor scalars and pseudo-scalars will behave differently among the set of observables.

Another remarkable point that becomes clear when comparing the set of clean observables $A_{T}^{(2)}, A_{T}^{(3)}$ and $A_{T}^{(4)}$ versus the old observables $F_{L}$ and $A_{F B}$ concerns the potential discovery of $\mathrm{NP}$, in particular of new right-handed currents. There are large deviations from the SM curve from the ones of the four supersymmetric benchmark points. A large deviation from the SM for $A_{T}^{(2)}, A_{T}^{(3)}$ or $A_{T}^{(4)}$ can thus show the presence of right-handed currents in a way that is not possible with $F_{L}$ or $A_{F B}$. In the latter cases the deviations from the SM prediction of the same four representative curves are marginal.

In the experimental plots we find a good agreement between the central values extracted from the fits and the theoretical input. Any deviations seen are small compared to the statistical uncertainties. The experimental resolution for $F_{L}$ is very good but with the small deviations from the SM expected this is not helpful in the discovery of new right-handed currents. Comparing the theoretical and experimental figures for the other observables it can be seen that in particular $A_{T}^{(3)}$ show great promise to distinguish between NP models.

Finally, let us mention that the old observables $F_{L}$ and $A_{F B}$ are already accessible to the BaBar [10,34] and Belle [35] experiments. The first measurements are shown in Fig. 6 with the SM predictions and the weighted SM averages over the bin $q^{2} \in\left[1 \mathrm{GeV}^{2}, 6 \mathrm{GeV}^{2}\right]$. All the present data is compatible with the SM predictions. For example, the first measurement of the Babar collaboration on $F_{L}$ in the low- $q^{2}$ region is given as an average over the bin $q^{2} \in$ $\left[4 m_{\mu}^{2}, 6.25 \mathrm{GeV}^{2}\right]$ :

$$
F_{L}\left(\left[4 m_{\mu}^{2}, 6.25 \mathrm{GeV}^{2}\right]\right)=0.35 \pm 0.16 \pm 0.04
$$

while the theoretical average, weighted over the rate, using the bin, $q^{2} \in\left[1 \mathrm{GeV}^{2}, 6 \mathrm{GeV}^{2}\right]$, based on our results is given by:

$$
F_{L}\left(\left[1 \mathrm{GeV}^{2}, 6 \mathrm{GeV}^{2}\right]\right)=0.86_{-0.05}^{+0.04} .
$$


Here, one should keep in mind that the spectrum below $1 \mathrm{GeV}^{2}$ is theoretically problematic due to the influence of very light resonances; moreover the rate and also the polarisation $F_{L}$ are changing dramatically around $1 \mathrm{GeV}^{2}$. Therefore, we strongly recommend to use the standard bin from $1 \mathrm{GeV}^{2}$ to $6 \mathrm{GeV}^{2}$ in all future measurements.

\section{Summary}

The full angular analysis of the decay $\bar{B}_{d} \rightarrow \bar{K}^{* 0} \mu^{+} \mu^{-}$at the LHC $b$ experiment offers great opportunities for the new physics search. New observables can be designed to be sensitive to a specific kind of NP operator within the model-independent analysis using the effective field theory approach. The new observables $A_{T}^{(2)}, A_{T}^{(3)}$ and $A_{T}^{(4)}$ are shown to be highly sensitive to right handed currents. Clearly, theoretical progress on the $\Lambda / m_{b}$ corrections would enhance their sensitivity significantly and would be highly desirable in view of a possible upgrade of the LHC $b$ experiment. Moreover, we have shown that the previously discussed angular distribution $A_{T}^{(1)}$ cannot be measured at either $\mathrm{LHC} b$ or at a Super- $B$ factory.

\section{Acknowledgement}

Support of the Eupopean network Heptools is acknowledged.

\section{Appendix}

We add here the explicit formula for the distribution functions and their symmetries:

In the massless limit, the distribution functions $I_{i}$ depend on products of the six complex $K^{*}$ spin amplitudes, $A_{\perp L / R}, A_{\| L / R}, A_{0 L / R}$ :

$$
\begin{aligned}
I_{1} & =\frac{3}{4}\left(\left|A_{\perp L}\right|^{2}+\left|A_{\| L}\right|^{2}+(L \rightarrow R)\right) \sin ^{2} \theta_{K}++\left(\left|A_{0 L}\right|^{2}+\left|A_{0 R}\right|^{2}\right) \cos ^{2} \theta_{K} \\
& \equiv a \sin ^{2} \theta_{K}+b \cos ^{2} \theta_{K}, \\
I_{2} & =\frac{1}{4}\left(\left|A_{\perp L}\right|^{2}+\left|A_{\| L}\right|^{2}\right) \sin ^{2} \theta_{K}+-\left|A_{0 L}\right|^{2} \cos ^{2} \theta_{K}+(L \rightarrow R) \\
& \equiv c \sin ^{2} \theta_{K}+d \cos ^{2} \theta_{K}, \\
I_{3} & =\frac{1}{2}\left[\left(\left|A_{\perp L}\right|^{2}-\left|A_{\| L}\right|^{2}\right) \sin ^{2} \theta_{K}+(L \rightarrow R)\right] \equiv e \sin ^{2} \theta_{K}, \\
I_{4} & =\frac{1}{\sqrt{2}}\left[\Re\left(A_{0 L} A_{\| L}^{*}\right) \sin 2 \theta_{K}+(L \rightarrow R)\right] \equiv f \sin 2 \theta_{K}, \\
I_{5} & =\sqrt{2}\left[\Re\left(A_{0 L} A_{\perp L}^{*}\right) \sin 2 \theta_{K}-(L \rightarrow R)\right] \equiv g \sin 2 \theta_{K}, \\
I_{6} & =2\left[\Re\left(A_{\| L} A_{\perp L}^{*}\right) \sin { }^{2} \theta_{K}-(L \rightarrow R)\right] \equiv h \sin { }^{2} \theta_{K}, \\
I_{7} & =\sqrt{2}\left[\Im\left(A_{0 L} A_{\| L}^{*}\right) \sin 2 \theta_{K}-(L \rightarrow R)\right] \equiv j \sin 2 \theta_{K}, \\
I_{8} & =\frac{1}{\sqrt{2}}\left[\Im\left(A_{0 L} A_{\perp L}^{*}\right) \sin 2 \theta_{K}+(L \rightarrow R)\right] \equiv k \sin 2 \theta_{K},
\end{aligned}
$$




$$
I_{9}=\left[\Im\left(A_{\| L}^{*} A_{\perp L}\right) \sin ^{2} \theta_{K}+(L \rightarrow R)\right] \equiv m \sin ^{2} \theta_{K}
$$

Taking into account $a=3 c$ and $b=-d$, we are left with 9 independent parameters which can be fixed experimentally in a full angular fit.

The distribution functions are invariant under the following three independent symmetry transformations of the spin amplitudes as one easily verifies, using the explicit formulae given above: (1) a global phase transformation of the $L$-amplitudes

$$
A_{\perp L}^{\prime}=e^{i \phi_{L}} A_{\perp L}, A_{\| L}^{\prime}=e^{i \phi_{L}} A_{\| L}, A_{0 L}^{\prime}=e^{i \phi_{L}} A_{0 L}
$$

(2) a global transformation of the $R$-amplitudes

$$
A_{\perp R}^{\prime}=e^{i \phi_{R}} A_{\perp R}, A_{\| R}^{\prime}=e^{i \phi_{R}} A_{\| R}, A_{0 R}^{\prime}=e^{i \phi_{R}} A_{0 R}
$$

and (3) a continuous $L \leftrightarrow R$ rotation

$$
\begin{gathered}
A_{\perp L}^{\prime}=+\cos \theta A_{\perp L}+\sin \theta A_{\perp R}^{*}, \quad A_{\perp R}^{\prime}=-\sin \theta A_{\perp L}^{*}+\cos \theta A_{\perp R} \\
A_{0 L}^{\prime}=+\cos \theta A_{0 L}-\sin \theta A_{0 R}^{*}, \quad A_{0 R}^{\prime}=+\sin \theta A_{0 L}^{*}+\cos \theta A_{0 R} \\
A_{\| L}^{\prime}=+\cos \theta A_{\| L}-\sin \theta A_{\| R}^{*}, \quad A_{\| R}^{\prime}=+\sin \theta A_{\| L}^{*}+\cos \theta A_{\| R} .
\end{gathered}
$$

\section{References}

[1] M. Artuso et al., arXiv:0801.1833 [hep-ph].

[2] T. Hurth, Rev. Mod. Phys. 75 (2003) 1159 [arXiv:hep-ph/0212304].

[3] T. Hurth, Int. J. Mod. Phys. A 22 (2007) 1781 [arXiv:hep-ph/0703226].

[4] U. Egede, T. Hurth, J. Matias, M. Ramon and W. Reece, arXiv:0807.2589 [hep-ph].

[5] A. Ishikawa et al. [Belle Collaboration], Phys. Rev. Lett. 91 (2003) 261601 [arXiv:hep-ex/0308044].

[6] B. Aubert et al. [BABAR Collaboration], Phys. Rev. Lett. 91 (2003) 221802 [arXiv:hep-ex/0308042].

[7] A. Ishikawa et al., Phys. Rev. Lett. 96 (2006) 251801 [arXiv:hep-ex/0603018].

[8] T. Hurth, G. Isidori, J. F. Kamenik and F. Mescia, arXiv:0807.5039 [hep-ph].

[9] B. Aubert et al. [BABAR Collaboration], Phys. Rev. D 73 (2006) 092001 [arXiv:hep-ex/0604007].

[10] B. Aubert et al. [BABAR Collaboration], arXiv:0804.4412 [hep-ex].

[11] J. Dickens, V. Gibson, C. Lazzeroni and M. Patel, CERN-LHCB-2007-038.

[12] J. Dickens, V. Gibson, C. Lazzeroni and M. Patel, CERN-LHCB-2007-039.

[13] U. Egede, CERN-LHCB-2007-057.

[14] T. Browder, M. Ciuchini, T. Gershon, M. Hazumi, T. Hurth, Y. Okada and A. Stocchi, JHEP 0802 (2008) 110 [arXiv:0710.3799 [hep-ph]].

[15] M. Bona et al., arXiv:0709.0451 [hep-ex].

[16] J. L. . Hewett et al., arXiv:hep-ph/0503261.

[17] A. G. Akeroyd et al. [SuperKEKB Physics Working Group], arXiv:hep-ex/0406071.

[18] M. Beneke, G. Buchalla, M. Neubert and C. T. Sachrajda, Phys. Rev. Lett. 83 (1999) 1914.

[19] C. W. Bauer, S. Fleming, D. Pirjol and I. W. Stewart, Phys. Rev. D 63 (2001) 114020 [arXiv:hep-ph/0011336].

[20] M. Beneke, A. P. Chapovsky, M. Diehl and T. Feldmann, Nucl. Phys. B 643 (2002) 431 [arXiv:hep-ph/0206152]. 
[21] J. Charles, A. Le Yaouanc, L. Oliver, O. Pène, and J.-C. Raynal, Phys. Rev. D 60, 014001 (1999); Phys. Lett. B 451, 187 (1999). See also M. J. Dugan and B. Grinstein, ibid. 255, 583 (1991).

[22] M. Beneke and T. Feldmann, Nucl. Phys. B 592 (2001) 3 [hep-ph/0008255].

[23] F. Krüger, Chapter 2.17 of Ref. [16].

[24] F. Kruger and J. Matias, Phys. Rev. D 71, 094009 (2005);

[25] E. Lunghi and J. Matias, JHEP 0704 (2007) 058 [arXiv:hep-ph/0612166].

[26] C. Bobeth, G. Hiller and G. Piranishvili, arXiv:0805.2525 [hep-ph].

[27] M. Beneke, T. Feldmann and D. Seidel, Nucl. Phys. B 612 (2001) 25 [arXiv:hep-ph/0106067]; Eur. Phys. J. C 41 (2005) 173 [arXiv:hep-ph/0412400].

[28] F. Krüger, L. M. Sehgal, N. Sinha, and R. Sinha, Phys. Rev. D 61, 114028 (2000); 63, 019901(E) (2001).

[29] D. Melikhov, N. Nikitin, and S. Simula, Phys. Lett. B 442, 381 (1998).

[30] C. S. Kim, Y. G. Kim, C.-D. Lü, and T. Morozumi, Phys. Rev. D 62, 034013 (2000).

[31] A. Faessler, T. Gutsche, M. A. Ivanov, J. G. Körner, and V. E. Lyubovitskij, EPJdirect C4, 18 (2002).

[32] A. Ali and A. S. Safir, Eur. Phys. J. C 25, 583 (2002).

[33] U. Egede,T. Hurth,J. Matias,M. Ramon and W. Reece, in preparation.

[34] Talk by K.Flood (BaBar collaboration) at ICHEP2008, see http://ichep08.com/ .

[35] Talk by J.-T.Wei (Belle collaboration) at ICHEP2008, see http://ichep08.com/ . 